\title{
Focal Acute Inflammation
}

National Cancer Institute

\section{Source}

National Cancer Institute. Focal Acute Inflammation. NCI Thesaurus. Code C82967.

An inflammatory process characterized by the localized collection of polymorphonuclear neutrophils. 